\title{
Confirmed inguinal lymphogranuloma venereum genovar L2c in a man who had sex with men, Slovenia, 2015
}

M Matičič ${ }^{1}$, I Klavs ${ }^{2}$, J Videčnik Zorman ${ }^{1}$, D Vidmar Vovko ${ }^{1}$, R Kogoj ${ }^{3}$, D Keše ${ }^{3}$

1. Clinic for Infectious Diseases and Febrile Illnesses, University Medical Centre Ljubljana, Ljubljana, Slovenia

2. Communicable Diseases Centre, National Institute of Public Health, Ljubljana, Slovenia

3. Institute of Microbiology and Immunology, Faculty of Medicine, University of Ljubljana, Ljubljana, Slovenia

Correspondence: Darja Keše (darja.kese@mf.uni-lj.si)

Matičič M, Klavs I, Videčnik Zorman J, Vidmar Vovko D, Kogoj R, Keše D. Confirmed inguinal lymphogranuloma venereum genovar L2c in a man who had sex with men, Slovenia, 2015. Euro Surveill. 2016;21(5):pii=30129. DOI: http://dx.doi.org/10.2807/1560-7917.ES.2016.21.5.30129

A laboratory-confirmed lymphogranuloma venereum (LGV) case in Slovenia was reported in 2015 , in a human immunodeficiency virus (HIV)-negative man presenting with inguinal lymphadenopathy. He reported unprotected insertive anal intercourse with two male partners in Croatia. Variant L2C of Chlamydia trachomatis was detected in clinical samples. Although the patient was eventually cured, the recommended treatment regimen with doxycycline had to be prolonged.

We describe a laboratory-confirmed case of lymphogranuloma venereum (LGV) in Slovenia, reported to the National Institute of Public Health (NIPH) in 2015 according to the Communicable Diseases Act that provides for mandatory universal reporting of all diagnosed LGV cases.

\section{Clinical case management}

In August 2015, a man who had sex with men in his late 405 with no medical history presented at the Clinic for Infectious Diseases and Febrile Illnesses, University Medical Centre Ljubljana, with a one-week history of painful swelling in the left groin, and sore throat. He reported no urethral discharge, no genital ulcers and no systemic symptoms. The baseline leukocytes and C-reactive protein (CRP) were normal and serology excluded infectious mononucleosis, toxoplasmosis and cat scratch disease. Fine needle aspiration tested negative for malignant cells. No antibiotics were prescribed. One week later he presented with fever $\left(38.5^{\circ} \mathrm{C}\right)$, malaise and unilateral inguinal erythema above the much increased swelling. Ultrasound of the left groin revealed two necrotic lymph nodes with abscess formation. Since LGV was suspected a bubo aspirate as well as urethral, pharyngeal and rectal swabs, and a urine sample were obtained for Chlamydia trachomatis (CT) DNA detection by real-time polymerase chain reaction (PCR). The urethral sample, bubo aspirate and urine tested positive for CT, meanwhile a real-time PCR specific for serovars of CT causing LGV (PCR LGV) tested positive for a urethral sample and a bubo aspirate. According to the 2013 European guideline on the management of LGV [1], doxycycline $100 \mathrm{mg}$ twice daily (bid) was prescribed for 21 days (Figure). Infections with Neisseria gonorrhoeae, human immunodeficiency virus (HIV), hepatitis B virus (HBV) and hepatitis C virus (HCV), Treponema pallidum, and urogenital mycoplasmas were excluded.

After five days of doxycycline, the patient's general condition improved, but the swelling increased and fluctuated with no spontaneous perforation, so a drainage incision of the swollen lymph node was performed, with pus testing positive by PCR LGV. Sixteen days after surgical incision, pus secretion from the incision wound still tested positive by PCR LGV, while culture yielded no $\mathrm{CT}$ isolates. The treatment with doxycycline was prolonged to 24 days by which time the incision wound was clean so the antibiotic therapy was stopped. However, two days later, the patient presented once more with painful erythematous swelling in the same area and abscess formation within necrotic lymph nodes, confirmed by ultrasound. Pus that was evacuated via a Penrose drain tested positive by PCR LGV, therefore the patient was again started on doxycycline. Despite the extended doxycycline treatment and drainage, the inguinal bubo did not subside and ultrasound examination revealed a new suppuration. Thirty-four days after the start of antibiotic therapy three necrotic lymph nodes were extirpated. The lymph node tissue proved positive in CT culture as well as by real-time PCRs for CT DNA and LGV-specific DNA. The doxycycline treatment was prolonged and subsequently stopped after clinical improvement, which occurred 54 days after initial administration. Six days later, the patient presented again with new buboes, 


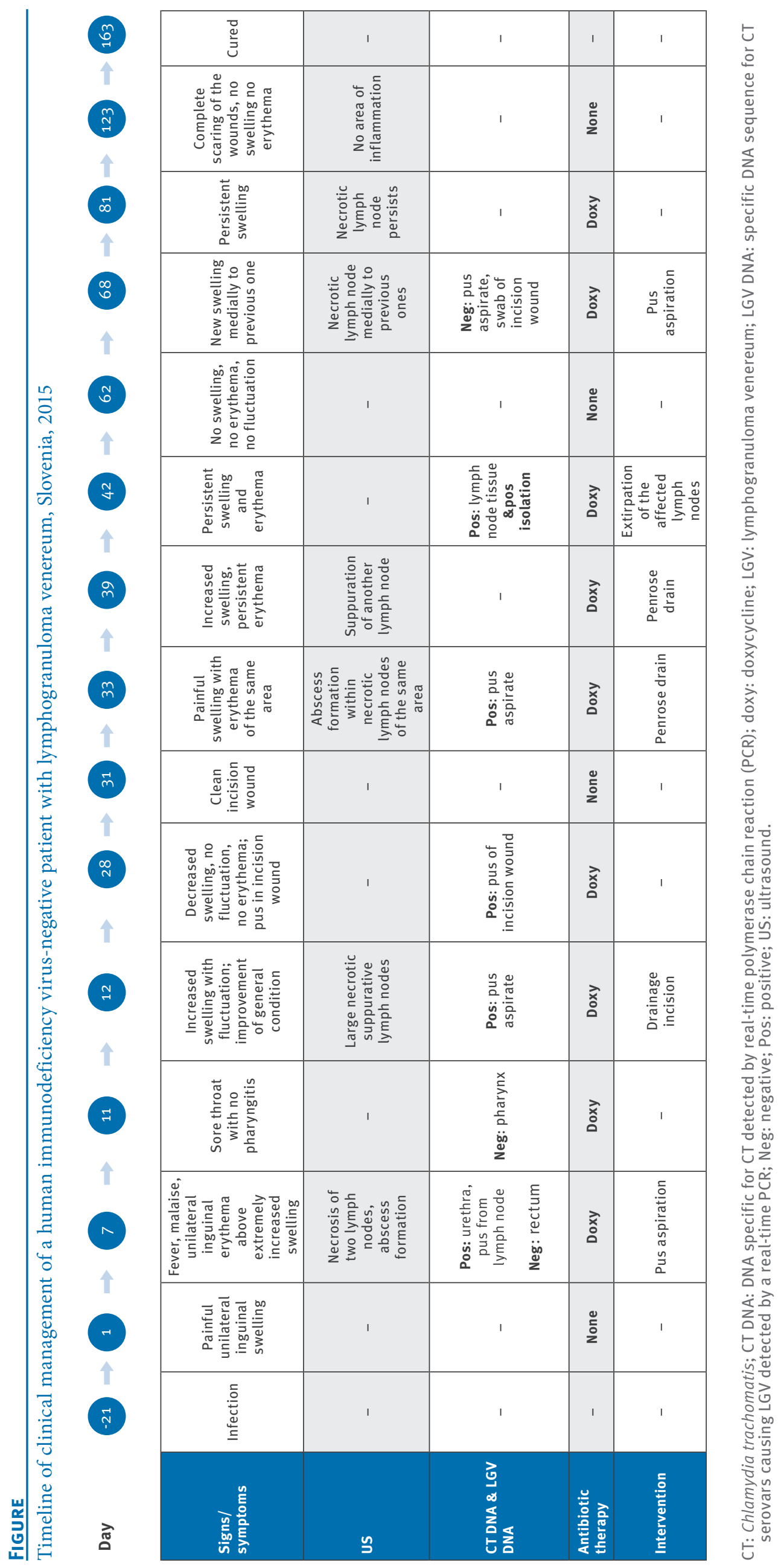


medially from the previous ones, and the ultrasound examination confirmed necrotic lymph nodes. Doxycycline was re-administered. Pus aspiration was negative for CT DNA and LGV-specific DNA by PCR. Clinical and ultrasound follow-up was continued on a two-week basis and the doxycycline treatment was stopped after 116 days, when clinical and ultrasound examination showed no remaining inflammation in the left inguinal region. On follow-up visit 40 days after latest treatment cessation no clinical relapse was noted.

\section{Laboratory confirmation}

Urethral, rectum and throat swabs, urine and pus aspirated from the suppurative lymph node, were tested for CT using COBAS TaqMan CT test, v2.o (Roche, Germany) according to manufacturer's instructions. All except rectum and throat swabs tested positive. Subsequently, LGV infection was identified by a realtime PCR using pmp-H gene specific primers together with a specific MGB probe for LGV biovar, including serovars L1, L2, L2b, L3 (Applied Biosystems, US), as described previously [2]. Sequencing of the ompA gene was performed to confirm LGV infection and to specify the biovar L strain. Analysing the obtained 1,003 bp double stranded consensus sequence by basic local alignment search tool (BLAST) algorithm showed a $100 \%$ match to the CT genotype variant L2C.

Lymph node tissue was cultured for 72 hour with monolayers of cycloheximide-treated McCoy cells. Only for one specimen were very small CT inclusions detected by using MOMP specific monoclonal antibodies conjugated with fluorescein (Trinity Biotech, Ireland).

Microimmunofluorescence test was performed for detection of CT IgG, IgA and IgM specific antibodies (FOCUS Diagnostics, US). High IgG $(1: 1,024)$ and IgA (1:128) titres were detected with CT specific antibodies. However, no IgM antibodies were found.

\section{Risk-behaviour and public health response}

Our patient reported sex with two male partners four weeks preceding the diagnosis, both of whom he had met on the same day through a mobile phone app. This occurred on the northern Croatian islands. One partner was a Croat, living in Germany, and the other a Slovenian, who had recently travelled to New York and to the coast of mid-western Africa. Our patient reported unprotected insertive anal and oral intercourse and no receptive anal intercourse, fisting or use of sex toys and no use of any drugs. He had no history of previous sexually transmitted infections (STI). The patient was counselled regarding prevention of other STI, including HIV and hepatitis C. Since the identity of contacts was not known, they could not be notified.

Information about the laboratory-confirmed LGV case in Slovenia was published in the last NIPH STI quarterly report. It was also included into the information about LGV in the 'Questions and Answers' format on the NIPH website as there have been no documented LGV cases in our country previously. Safer sex promotion, including condom use promotion, as well as promotion of seeking healthcare when having signs and symptoms of any STI among men who have sex with men (MSM) has been ongoing within the framework of the National strategy for HIV prevention and control according to plans and allocated resources. Information about the case was also forwarded to the three key Slovenian MSM non-governmental organisations for possible dissemination through their communication channels.

\section{Background}

Lymphogranuloma venereum is caused by CT strains of serovars L1, L2 and L3. Since 2003 several European countries reported a series of LGV outbreaks among the population of MSM [3]. These cases mostly presented with proctitis, caused by $L 2 b$ variant and the majority of these MSM were co-infected with HIV and other STI $[1,3]$. In 2010 , a case of LGV in a MSM was detected in a central European country, the Czech Republic [4]. Together with Hungary, these are the only two central European countries that reported LGV cases to the European Centre for Prevention and Control of Disease (ECDC) by the end of 2013 [5]. The number of reported LGV cases to ECDC underestimate true LGV incidence, since many countries do not routinely report LGV cases and because genotyping, which is necessary to confirm cases, is not always available [5].

\section{Discussion}

The laboratory-confirmed LGV case reported here was different from the majority of LGV cases in Europe $[3,5]$. It occurred in a HIV-negative MSM who presented with inguinal lymphadenopathy and who was infected with CT genotype variant $\mathrm{L} 2 \mathrm{C}$. The infection proved difficult to treat with doxycycline according to the current European guidelines [1].

Indeed, in contrast to our case, the majority of LGV cases reported in Europe occur in HIV-positive individuals [3,5]. In 2013, information on HIV status was available for 520 LGV cases (50\%) reported to ECDC, of whom $62 \%$ occurred in HIV-positive individuals and only $14 \%$ in HIV-negative (for $24 \%$ HIV status was unknown) [5].

Moreover, while most LGV cases among MSM in Europe present as severe proctitis our case presented as inguinal lymphadenopathy [3].The diagnosis of LGV can easily be missed in the first stages of infection as well as with overt clinical signs, especially, if it is uncommon in a particular geographic area. Knowledge of high-risk sexual behaviours of a patient may be crucial for correct diagnosis. The differential diagnosis of inguinal lymphadenopathy must include LGV, particularly in MSM.

As opposed to our case where disease was caused by CT genotype variant L2C, most LGV cases among MSM in Europe are infected with CT serovar L2b [1]. Although cases of LGV caused by this new LGV L2C variant, that originates from a recombination of $L 2$ and $D$ strains of 
$\mathrm{CT}$, have been reported, they all clinically manifested as proctitis and not as inguinal lymphadenopathy $[6,7]$.

In addition, our patient did not respond to treatment with doxycycline $100 \mathrm{mg}$ bid for 21 days, according to the 2013 European guideline on the management of lymphogranuloma venereum [1]. Eventually, prolonged treatment with doxycycline (109 days), drainage of pus and extirpation of necrotic lymph nodes resulted in cure. Several studies have shown the failure of the recommended treatment regimen $[8,9]$. The new LGV variant $L 2 C$ with inguinal clinical presentation might be more aggressive compared with the variant $L 2 b$, as it also was present in the anorectal region. Thus this may require a different treatment approach with extended doxycycline regimen or new antimicrobial options [7]. Revision of the current clinical guideline on the management of LGV, possibly distinguishing between the two variants, could be considered.

In Slovenia the national guidelines for treatment of STIS follow the European STI treatment guidelines [10], yet taking into account the national particularities. Careful clinical and microbiological monitoring of future LGV cases within the network of STI outpatient services will be crucial in evaluating any need to change the current LGV treatment guidelines. Since in Slovenia clinicians treating MSM with signs and symptoms of possible LGV rarely demand confirmation of LGV infection even when CT has been confirmed in a clinical specimen, the occurrence of LGV among Slovenian MSM is most likely underestimated. Although there exists laboratory capacity to diagnose LGV, the Institute of Microbiology and Immunology, Faculty for Medicine, University of Ljubljana that performs the vast majority of STI tests in Slovenia, reported only five LGV test requests in 2015. In addition to the notification of the presented laboratory-confirmed LGV case in Slovenia, in 2015 the NIPH received also a notification of three suspected $L G V$ cases that occurred in HIV-positive MSM, all presenting with clinical signs and symptoms consistent with LGV, including proctitis. Being managed at a private medical facility they did not wish the suggested microbiological testing (in one case CT infection was confirmed) because this would incur additional expenses to them, therefore they opted for empirical treatment. Their symptoms resolved after administration of doxycycline $100 \mathrm{mg}$ bid for 21 days.

Enhanced awareness of LGV together with promoting national LGV testing might unveil a hidden LGV epidemic among MSM in Slovenia. The proposed diagnostic algorithm for any MSM presenting with clinical signs and symptoms suggesting a possible LGV and a laboratory-confirmed CT infection, includes LGV confirmation at any of the STI outpatient services. However, additional resources are required for its realisation. Similar approaches in other central European countries with no cases reported to ECDC up to date might unveil hidden LGV epidemics among MSM.
Acknowledgements

The authors would like to express their gratitude to Sabina Pervić for her technical help.

\section{Conflict of interest}

None declared.

\section{Authors' contributions}

$M M$, IK and DK wrote the manuscript together. MM, JVZ, DVV treated the patient. DK, RK performed microbiological testing.

\section{References}

1. de Vries HJC, Zingoni A, Kreuter A, Moi H, White JA, European Branch of the International Union against Sexually Transmitted Infections, et al. 2013 European guideline on the management of lymphogranuloma venereum.J Eur Acad Dermatol Venereol. 2015;29(1):1-6. DOI: 10.1111/jdv.12461 PMID: 24661352

2. Morré SA, Spaargaren J, Fennema IS, de Vries HJ, Coutinho RA, Peña AS. Real-time polymerase chain reaction to diagnose lymphogranuloma venereum. Emerg Infect Dis. 2005;11(8):13112. DOI: 10.3201/eid1108.050535 PMID: 16110579

3. Savage EJ, van de Laar MJ, Gallay A, van der Sande M, Hamouda 0 , Sasse A, et al. Lymphogranuloma venereum in Europe, 2003-2008. Euro Surveill. 2009;14(48):19428.PMID: 20003898

4. Vanousova D, Zakoucka H, Jilich D, Rozsypal H, Stankova M, Zufanova S, et al. First detection of Chlamydia trachomatis LGV biovar in the Czech Republic, 2010-2011. Euro Surveill. 2012;17(2):20055.PMID: 22264863

5. European Centre for Disease Prevention and Control (ECDC). Sexually transmitted infections in Europe 2013. Stockholm: ECDC; 2015. Available from: http://ecdc.europa.eu/en/ publications/Publications/sexual-transmitted-infectionseurope-surveillance-report-2013.pdf

6. Stary G, Meyer T, Bangert C, Kohrgruber N, Gmeinhart B, Kirnbauer R, et al. New Chlamydia trachomatis L2 strains identified in a recent outbreak of lymphogranuloma venereum in Vienna, Austria. Sex Transm Dis. 2008;35(4):377-82. DOI: 10.1097/OLQ.ob013e31815d6df8 PMID: 18209688

7. Somboonna N, Wan R, Ojcius DM, Pettengill MA, Joseph SJ, Chang A, et al. Hypervirulent Chlamydia trachomatis clinical strain is a recombinant between lymphogranuloma venereum (L(2)) and D lineages. MBio. 2011;2(3):e00045-11. DOI: 10.1128/ mBio.00045-11 PMID: 21540364

8. Méchaï F, de Barbeyrac B, Aoun O, Mérens A, Imbert P, Rapp C. Doxycycline failure in lymphogranuloma venereum. Sex Transm Infect. 2010;86(4):278-9. DOI: 10.1136/sti.2009.042093 PMID: 20660591

9. Oud EV, de Vrieze NH, de Meij A, de Vries HJ. Pitfalls in the diagnosis and management of inguinal lymphogranuloma venereum: important lessons from a case series.Sex Transm Infect. 2014;90(4):279-82. DOI: 10.1136/sextrans-2013-051427 PMID: 24787368

10. The International Union against Sexually Transmitted Infections (IUSTI). Available from http://www.iusti.org/ regions/europe/euroguidelines.htm

\section{License and copyright}

This is an open-access article distributed under the terms of the Creative Commons Attribution (CC BY 4.0) Licence. You may share and adapt the material, but must give appropriate credit to the source, provide a link to the licence, and indicate if changes were made.

This article is copyright of the authors, 2016. 